\title{
REAL COMMUTATIVE SEMIGROUPS ON THE PLANE. II $\left({ }^{1}\right)$
}

BY

\author{
J. G. HORNE, JR.
}

1. In the first note of this title, a study was begun of commutative topological semigroups on the plane $E$ which contain an appropriate copy of the multiplicative semigroup $R$ of all real numbers. Among other things, the product semigroup on $R \times R$ was distinguished from among all such. The main result of this note is to shift commutativity and a part of the assumption concerning $R$ from the hypothesis to the conclusion of various theorems. Thus, in spite of the title, we do not assume commutativity, and the assumption concerning $R$ is considerably weakened. On the other hand, these properties are never far away in our present study. In particular, a characterization of $R \times R$ is given under these weaker conditions.

The assumption which replaces that about $R$ is simply that $E$ contains a zero 0 which belongs to the closure of the component $G$ of the identity of the maximal subgroup $H(1)$. The other assumptions which are necessary for the final result anyway force the presence of a copy of $R$. Actually even this hypothesis is suspect. We have some reason for thinking that the assumption about square roots of 1 (see the main theorem) leads to the presence of a zero and if $H(1)$ is dense in $E$ there seems to be some hope in showing $0 \in G^{-}$. We expect to say more about this later.

2. In this section we collect the definition of various symbols and terms and list certain of our assumptions.

First, $E$ will consistently denote a topological semigroup on the plane with zero 0 and identity 1 . These elements are characterized by the identities: $1 \cdot x=x \cdot 1=x$ and $0 \cdot x=x \cdot 0=0$ for all $x \in E$. For $x \in E$, we set $x^{2}=x \cdot x$ and say that an element $x$ is nilpotent if $x \neq 0$ and $x^{2}=0$ (this requires more than is usually required, but is satisfactory for our purposes). An element $x$ is a square root of 1 if $x^{2}=1$. An element $x$ is an idempotent if $x^{2}=x$.

For sets $X, Y \subset E, X-Y$ denotes the complement of $Y$ in $X, X Y$ denotes the set of all products $x y$ with $x \in X, y \in Y$, and $X^{-}$denotes the closure of $X$ in $E$.

By an isomorphism between two topological semigroups $S$ and $T$ is meant a function from $S$ onto $T$ which is both a topological and algebraic isomorphism. Of course, $S$ and $T$ are said to be isomorphic if such an isomorphism exists, a fact which we signify by writing $S \cong T$.

Following the usage in [4] and elsewhere, $H(1)$ denotes the maximal sub-

Presented to the Society, January 23, 1961; received by the editors August 16, 1961.

(1) Work on this paper was supported in part by Contract NSF-G17847. 
group of $E$, i.e. the set of all $x$ in $E$ which have an inverse with respect to 1 . The component of the identity in $H(1)$ is denoted by $G$ and the boundary of $G$ by $L$. We invariably use $R$ to denote the multiplicative semigroup of all real numbers.

In addition to the above somewhat more natural assumptions about $E$ we now list the following which forms a standing assumption throughout the paper:

$E$ contains no nilpotent elements and 0 belongs to the closure of $G$.

As we have implied, the joining of these two hypotheses in one does not reflect our feelings about their nature and is done simply for the sake of brevity.

3. This section begins with a statement of the main theorem and then proceeds to establish a number of results, listed as propositions, on which its proof is based. Several of these results are analogous to results in [1] and in some cases we simply indicate the modifications necessary to make proofs there adequate here. The proof of the main theorem is postponed until these propositions are out of the way. The paper concludes with two remarks about the hypotheses (2) and (3).

MaIn Theorem. Suppose E is a topological semigroup on the plane with zero 0 and identity 1 . Assume that $0 \in G^{-}$and that $E$ contains no nilpotent elements. Then the following conditions are equivalent:

(1) $E \cong R \times R$,

(2) E has exactly four idempotents and 1 has at least three square roots,

(3) $H(1)$ is dense in $E$ and has at least three components.

According to [4], $H(1)$ and $G$ are Lie groups which are open subsets of the plane and $L$ is an ideal in $G \cup L$. Since $G$ is connected, it is (topologically) the cartesian product of a compact subgroup and a Euclidean space (see [7], for example). If the compact group is nontrivial it is the circle group, $G$ is isomorphic to the multiplicative group of nonzero complex numbers and $H(1)=G[3]$. If the compact group is trivial then $G$ is topologically a plane and hence is either the two dimensional vector group or the group of affine transformations of the line [6, pp. 258, 238 and 257].

Thus each of conditions (2) and (3) force $G$ to be one of the latter two groups. The possibilities for $L$ in this case were determined in [5]. The only case in which $L$ contains a zero and $G^{-}$contains no nilpotent elements is the case in which $G$ (and therefore $G^{-}$) is actually commutative, $L$ is a line and $L-\{0\}$ is the union of two groups $A$ and $B$ with $A B=\{0\}$.

According to [2], whenever $G$ is one of the groups on the plane, there is a one-parameter subgroup $P \subset G$ emanating from every right zero in $L$. Thus under the present circumstances, if either (2) or (3) holds, there exists a one-parameter subgroup $P \subset G$ such that $P^{-}=P \cup\{0\}$. The argument given in [1] is now adequate with almost no change to show that $G^{-} \cong P^{-} \times P^{-}$. We thus have the following result: 
Proposition 1. If either condition (2) or (3) holds then there exists a oneparameter subgroup $P \subset G$ with $P^{-}=P \cup\{0\}$ and $G^{-}$is isomorphic to $P^{-} \times P^{-}$.

For the remainder of the paper we take a fixed one-parameter subgroup of $G$ having the properties just given. All reference to $P$ is to this subgroup.

Proposition 2. If $G^{-}$is isomorphic to $P^{-} \times P^{-}$then $H(1)$ has only a finite numbers of components.

Proof. The proof of this result parallels and uses parts of the proof of the corresponding result in [1]. Suppose $H(1)$ contains an infinite sequence of components $C_{1}, C_{2}, \cdots$. Each $C_{i}$ has the form $x_{i} G$ for some $x_{i} \in H(1)$. Since the boundary $L$ of $G$ is a (closed) topological line and multiplication by $x_{i}$ is seen to be a homeomorphism on $E$, each $x_{i} G$ is topologically a plane whose boundary is a closed topological line. Furthermore, $0 \in\left(x_{i} G\right)^{-}$for all $i$. Let $S$ be a circle with center at 0 . Recall that $A$ denotes one of the components of $L-\{0\}$. For each $i$ we can choose a largest point $s_{i}$ of $x_{i} A$ so that $x_{i} \in S$. There is then a point $x \in S$ which is a cluster point of the $s_{i}$. It is then not difficult to show, as in [1], that $x$ is also a cluster point for a sequence of points each belonging to some $x_{i} B$ where $B$ is the other component of $L-\{0\}$. Thus we can construct two sequences of points which we may as well call $s_{i}$ and $t_{i}$ so that $s_{i} \rightarrow x, t_{i} \rightarrow x, s_{i} \in x_{i} A$ and $t_{i} \in x_{i} B$. If we can show that $x_{i} B=B x_{i}$ we have $s_{i} t_{i}=0$ for all $i$ since $A B=\{0\}$. Therefore $x^{2}=0$ and we have a nilpotent element, contrary to our standing assumption. Since $x_{i} G=G x_{i}$ we have $x_{i} L=L x_{i}$. The only question then is whether $x_{i} b=a x_{i}$ is possible for some $b \in B, a \in A$. But if so then $\left(x_{i} b\right)\left(x_{i} b\right)=\left(x_{i} b\right)\left(a x_{i}\right)=x_{i}(b a) x_{i}=0$ which again is contrary to our assumption about nilpotent elements. Thus $x_{i} B$ $=B x_{i}$ and the proof of the proposition is complete.

Assume that $G^{-}$is isomorphic to $P^{-} \times P^{-}$. By the previous result, $H(1)$ has a finite number of components. Let $C_{0}=G$ and let $C_{1}, \cdots, C_{n}$ denote the remaining components of $H(1)$. Take $x_{i} \in C_{i}$; thus $C_{i}=x_{i} G$. We now develop some notation and terms for use in the next proposition and again in Proposition 7. This is done in lieu of talking about rays and sectors as was done in [1] in proving that $H(1)$ has at most four components.

Since the components $A$ and $B$ of $L-\{0\}$ are orbits under the action of $G, x_{i} A$ and $x_{i} B$ are orbits under the action of $G$ for any $x_{i}$. Thus if for any $i, j,\left(x_{i} A\right) \cap\left(x_{j} A\right) \neq \varnothing$ then $x_{i} A=x_{j} A$. A similar statement holds for the $x_{i} B$. Since $x_{i} B=B x_{i},\left(x_{i} A\right) \cap\left(x_{j} B\right)=\varnothing$ for all $i, j$. Now each $C_{i}^{-}$is a closed halfplane which contains 0 . If a distinct pair $C_{\bar{i}}$ and $C_{\bar{j}}$ have a nonzero point in common it is a boundary point and they have an entire edge (either $x_{i} A^{-}$ or $\left.x_{i} B^{-}\right)$in common. Thus $E-H(1)$ is a set which is separated by the omission of zero into $n+1$ components $D_{0}, \cdots, D_{n}$. Furthermore, each set $D_{\vec{i}}$ is either a closed half-line or a closed half-plane. Number the $C_{i}$ in a counterclockwise direction from $G$ and assume that $D_{i}$ is between $C_{i}$ and $C_{i+1}$ (in the obvious sense) for $i=0,1, \cdots, n-1$, and $D_{n}$ is between $C_{n}$ and $C_{0}$. We shall refer to $C_{1}$ as the first and $C_{n}$ as the last component of $H(1)$ after $G$. 
Proposition 3. Suppose $G^{-}=P^{-} \times P^{-}$. If $C$ is either the first or last component of $H(1)$ then there exists $x \in C$ with $x^{2}=1$.

Proof. Let $\chi(x)=x^{2}$ for $x \in E$. For each $i, \chi\left(C_{i}\right)=C_{j}$ for some $j$ and $j=i$ if and only if $i=0$. Also, since there are no nilpotent elements, $\chi\left(D_{i}\right) \subset D_{j}$ for each $i$ and some $j$. Assume $C=C_{1}$. The argument is similar if $C=C_{n}$. Now $D_{0}$ has a nonzero point in common with $L$ and hence contains one of the components of $L-\{0\}$. Let this component be $A$. Since $\chi(A) \subset A, \chi\left(D_{0}\right)$ $\subset D_{0}$.

Now $\chi(C)$ must have nonzero boundary points in common with $\chi\left(D_{0}\right)$, $\chi(C)$ is a component of $H(1)$ and $\chi(C) \neq C$. Since $\chi\left(D_{0}\right) \subset D_{0}$, the only component of $H(1)$ satisfying these conditions is $G$. Hence $\chi(C)=G$, so there exists $x \in C$ such that $x^{2}=1$.

Proposition 4. Assume $G^{-}=P^{-} \times P^{-}$. If $x^{2}=1$ then $x$ is in the center of $E$; i.e. $x y=y x$ for all $y \in E$.

Proof. Let $g(y)=x y x$ for $y \in E$. Let $F$ denote the fixed-point set of $g$, that is, $F$ is the set of $y$ such that $g(y)=y$. Obviously $g$ is an involution on $E$ so $F$ is either a single point, a closed line or all of $E$. Now $G$ is a normal subgroup so $g(G) \subset G$. Hence $g(L) \subset L$. Let $A$ and $B$ be the two components of $L-\{0\}$; let $e$ be the idempotent in $A$ and $f$ the idempotent in $B$. Now $(g(e))^{2}=(x e x)^{2}$ $=(x e x)(x e x)=x e x=g(e)$ so $g(e)$ is a (nonzero) idempotent in $L$. If $g(e)=f$ then $x e x=f$ so $x e=f x$. But we have seen that $x A \cap B x=\varnothing$ for all $x \in H(1)$ so this is impossible. Therefore $x e x=e$. Similarly $x f x=f$. Thus $g$ induces an involution on $L$ with two fixed points so $L \subset F$. Since $1 \in F$ we infer from the above list of possibilities for $F$ that $F=E$. Hence $x y=y x$ for all $y \in E$.

Proposition 5. Suppose $x \neq 0, P x=x P$ and $P x$ is a sub-semigroup of $E$. Then $P x$ is a group isomorphic to $P$.

Proof. The proofs of Lemma 1.1 and Theorem 1.2 of [1] apply without change here to show that the map $t \rightarrow t x$ is a homeomorphism from $P^{-}$to $P^{-} x$ (even more: for future reference we mention that $P^{-} x$ is a closed subset of $E$ ). The same is true for the map $t \rightarrow x t$. Therefore for each $t \in P$ there is a unique element $h(t) \in P$ such that $t x=x h(t)$. It is easy to see that $h$ is a continuous function from $P$ to itself and furthermore, since

$$
(s t) x=s(t x)=s(x h(t))=(s x) h(t)=(x h(s)) h(t)
$$

and also

$$
(s t) x=x h(s t),
$$

we have $h(s t)=h(s) h(t)$ for all $s, t \in P$. Therefore there exists $u \in P$ such that $h(t)=t^{u}$; i.e. $t x=x t^{u}$ for all $t \in P$. Since $x^{2} \in P x$ we have $x^{2}=p x$ for some $p \in P$. Now $x p x=x\left(x p^{u}\right)=x^{2} p^{u}=(p x) p^{u}=p\left(x p^{u}\right)=p^{2} x$. Also, $x p x=(x p) x=\left(p^{1 / u} x\right)$ 
$=p^{1 / u} x^{2}=p^{1 / u} p x$. Therefore $p^{2} x=p^{1 / u} p x$. Hence $p^{2}=p^{1 / u} p$ so $p=p^{1 / u}$. Thus, either $p=1$ and $x$ is an idempotent or $u=1$. In the latter case $t x=x t$ for all $t \in P$. In this case $x / p$ is an idempotent. In any case, $P x$ contains an idempotent $e$. Since $P e=P x$ and $e P=x P$, it follows that $P e=e P$ so $e$ is an identity for $P e$ and hence $P x$. Thus $P x$ is a semigroup with identity and no other idempotents so $P x$ is a group isomorphic to $P$.

Proposition 6. Suppose $G^{-}=P^{-} \times P^{-}$. Then 1 has at least three square roots if and only if $H(1)$ has at least three components. In either case, 1 has exactly four square roots, $H(1)$ has exactly four components and $H(1)$ is the product of $G$ and the four group. In particular, $H(1)$ is commutative.

Proof. If $x \neq y$ and $x^{2}=y^{2}=1$ then $x G \neq y G$. Therefore if 1 has at least three square roots then $H(1)$ has at least three components. The converse is an immediate consequence of Proposition 3.

Now suppose 1 has at least three square roots. By Proposition 4 , if $x^{2}=1$ then $x$ is in the center of $G$. The knowledge that if $x^{2}=1$ then $x y=y x$ for $y \in E$ is all that is needed to make the argument given in [1] applicable under the present circumstances to show that $H(1)$ has exactly four components. Of course each component contains a square root of 1 so the remainder of the proposition follows immediately.

Proposition 7. Assume $G^{-}=P^{-} \times P^{-}$. Let $x_{1}, x_{2}, x_{3}$ and 1 be distinct square roots of 1 . Let the fixed point set of the mapping $x \rightarrow x x_{i}$ be denoted $F_{i}$ for $i=1,2,3$. Then we may assume the $x_{i}$ numbered so that $F_{1}$ and $F_{3}$ are (closed) semigroups isomorphic to $R$ and $F_{1} \cap F_{3}=F_{2}=\{0\}$.

Proof. Let $V=\left\{x_{1}, x_{2}, x_{3}, 1\right\}$. Then $V$ is the four group which obviously acts effectively on $E$. It is well known such an action is equivalent to the ordinary action. (See [8], for example.) Therefore, two of the fixed-point sets are closed lines and the third is the point of intersection of these two. There is no harm in labeling the first two $F_{1}$ and $F_{3}$ and the third $F_{2}$. Since $x_{1}$ is in the center of $E, F_{1}$ is an ideal in $E$ and in particular a sub-semigroup of $E$. A corresponding statement holds for $F_{3}$. Since $F_{1} \cap F_{3}=F_{2}$ and $F_{2}$ is an ideal containing but one point, $F_{2}=\{0\}$.

Now multiplication by $x_{1}$ reflects $E$ about $F_{1}$, so $F_{1}$ separates $G$ and $x_{1} G$. We now use the notation and language developed just prior to Proposition 3. Suppose $x_{i}$ is the member of $V$ such that $x_{i} G$ is the first component of $H(1)$ after $G$. Then $x_{i} D_{0} \subset D_{0}$. There are now two possibilities. Either $D_{0}$ is one of the components of $L-\{0\}$ or the interior of $D_{0}$ is a plane. In either case, since $x_{i} D_{0} \subset D_{0}$ and an involution on a plane or a line has at least one fixed point, $D_{0}$ contains a nonzero fixed point under multiplication by $x_{i}$. Thus either $F_{1}$ or $F_{3}$ passes through $D_{0}$. There is nothing lost in assuming it is $F_{1}$. Let $x \in F_{1} \cap D_{0}$. Certainly $G D_{0} \subset D_{0}$ and $D_{0} G \subset D_{0}$ so $P x \subset D_{0}$ and $x P \subset D_{0}$. Since $F_{1}$ is an ideal, $P x$ and $x P$ are contained in $F_{1}$. As we know, 
$P^{-} x$ is a closed homeomorph of $P^{-}$so since $F_{1}$ is a line, $P x=x P$. It is clearly impossible for $F_{1} \cap D_{0}$ to contain points outside $P x$. Since $x^{2} \in F_{1} \cap D_{0}$, $x^{2} \in P x$. Since $P x=x P$ this implies $P x$ is a sub-semigroup of $E$. By Proposition 5, $P x$ is a group isomorphic to $P$. That $F_{1}$ is isomorphic to $R$ now follows easily since $x_{2}$ induces a reflection on $F_{1}$ whose only fixed point is 0 . The argument for $F_{3}$ is similar so the proof of the proposition is complete.

Proof of the Main Theorem. We prove $(2) \Rightarrow(3) \Rightarrow(1)$. It is obvious that $(1) \Rightarrow(2)$.

Assume (2); according to Proposition 6, 1 has four square roots and $H(1)$ has exactly four components. Thus we have the second part of (3). Now denote the square roots of 1 by $1, x_{1}, x_{2}$ and $x_{3}$ and denote the fixed-point set of the mapping $x \rightarrow x x_{i}$ by $F_{i}$ for $i=1,2,3$. By Proposition 7 we may suppose $F_{1}$ and $F_{2}$ are ideals in $E$, each isomorphic to $R$ and $F_{1} \cap F_{2}=F_{3}=\{0\}$. Thus there are distinct nonzero idempotents $f_{1} \in F_{1}$ and $f_{2} \in F_{3}$. By Proposition 1 , $G^{-}=P^{-} \times P^{-}$so $G^{-}$has four idempotents. If $E$ has only four idempotents then $f_{1}, f_{3} \in L$. Thus $L=P^{-} f_{1} \cup P^{-} f_{3}$. This means that multiplication by $x_{1}$ simply reflects $G$ about $P^{-} f_{1}$ so $x_{1} G$ has $P^{-} f_{1}$ in its boundary. In a similar manner we see that $x_{3} G$ has $P^{-} f_{3}$ in its boundary. Now the boundary of $x_{2} G$ is $x_{2} L=x_{2} P^{--} f_{1} \cup x_{2} P^{-} f_{3}$. Furthermore, multiplication by $x_{2}$ induces reflection in $F_{i}$ about 0 for $i=1,3$, so the boundary of $x_{2} G$ is the complement in $F_{1} \cup F_{3}$ of $x_{2}(L-\{0\})$. Thus $x_{2} G$ is all of the complement of $\left(G \cup x_{1} G \cup x_{3} G\right)^{-}$. Therefore, $H(1)$ is dense in $E$ and we have that (2) implies (3).

Now assume (3). Then $G^{-}=P^{-} \times P^{-}$so by Proposition 6,1 has exactly four square roots and $H(1)$ is a commutative group. Since $H(1)$ is dense in $E, E$ is a commutative semigroup. Also, according to Proposition 7 , we can choose an element $x_{2}$ so that $\left(x_{2}\right)^{2}=1$ and $x \neq 0$ implies $x_{2} x \neq x$. It is now straightforward to prove $E=R \times R$. Alternately, observe that $P^{-} \cup P x_{2}$ is a semigroup through the zero and identity of $E$ which is isomorphic to $R$. Therefore $E$ is a real commutative semigroup. With $x_{2}$ playing the role of -1 we see that $E \cong R \times R$ now follows from Theorem 5.2 of [1].

Two final remarks are in order. First, examples in [1] show that it is not possible to assume fewer than three square roots of the identity in (2), nor fewer than three components for $H(1)$ in (3) without making the theorem false.

Second, in virtue of Proposition 6, a certain amount of mixing of the hypotheses in (2) and (3) is possible without affecting the validity of the theorem. Specifically, the assumption concerning idempotents in (2) is interchangeable with the assumption concerning the denseness of $H(1)$ in (3). Furthermore, the hypothesis concerning square roots of 1 in (2) is interchangeable with that concerning components of $H(1)$ in (3). For after an interchange of the first sort we can still conclude that $G^{-}=P^{-} \times P^{-}$. Therefore Proposition 6 is applicable so the assumption concerning square roots is 
seen to be equivalent to the assumption concerning the number of components of $H(1)$.

\section{BIBLIOGRAPHY}

1. J. G. Horne, Jr., Real commutative semigroups on the plane, Pacific J. Math. 11 (1961), 981-997.

2. - On one parameter subgroups in semigroups in the plane, Michigan Math. J. (to appear).

3. P. S. Mostert and A. L. Shields, On a class of semigroups on $E_{n}$, Proc. Amer. Math. Soc. 7 (1956), 729-734.

4. - Semigroups with identity on a manifold, Trans. Amer. Math. Soc. 91 (1959), 380-389.

5. P. S. Mostert, Plane semigroups, to appear.

6. L. Pontrjagin, Topological groups, Princeton Univ. Press, Princeton, N. J., 1946.

7. Hans Samelson, Topology of Lie groups, Bull. Amer. Math. Soc. 58 (1952), 2-37.

8. P. A. Smith, New results and old problems in finite transformation groups, Bull. Amer. Math. Soc. 66 (1960), 401-415.

UNIVERSITY OF GEORGIA, Athens, Georgia 